\title{
Pattern Size Effects on Demolding Force for Imprint Process
}

\author{
Norihiro Fujikawa, Hiroaki Kawata, Masaaki Yasuda and Yoshihiko Hirai \\ Physics and Electronics, Graduate School of Engineering \\ Osaka Prefecture University, Sakai 599-8531, Japan \\ CREST-JST, Kawaguchi, Saitama 332-0012, Japan
}

\begin{abstract}
The demplding forces for thermal imprint to polystyrene film are studied. Two molds with $0.4 \mu \mathrm{m}$ and $4 \mu \mathrm{m}$ pitch LS patterns are fabricated. The pattern heights are adjusted in order to keep the side wall areas of both the molds same. The demolding force for the $0.4 \mu \mathrm{m}$ mold is much smaller than that for the $4 \mu \mathrm{m}$ mold. The pattern height rather than the side wall area is important factor for the demolding force. The demolding force is increased by the $\mathrm{UV} / \mathrm{O}_{3}$ treatment. The effect is more obvious for the $0.4 \mu \mathrm{m}$ mold than for the $4 \mu \mathrm{m}$ mold, because the pattern density of the $0.4 \mu \mathrm{m}$ mold is larger than that of the $4 \mu \mathrm{m}$
\end{abstract}

Keywords: imprint, demolding force, adhesion, friction, Si mold

\section{Introduction}

Nanoimprint lithography (NIL) is very powerful technology for nanofabrication because very fine pattern can be obtained by relatively low cost machine [1]. However, there still remain some issues. One of the significant issues is demolding. Since a mold usually adheres to resist polymer after molding, it has to be mechanically released from the resist. Fatal pattern defects are sometimes induced in the demolding process. Thus, it is very important to examine the demolding force $[2,3]$. The demolding force consists of the adhesion and friction forces. The adhesion force works at the top and bottom surfaces of the mold pattern and both the adhesion and friction forces work at the side wall as shown in Fig. 1. It has been already shown that the demolding force is proportional to the side wall area [4]. However, since the constant pitch pattern of $4 \mu \mathrm{m}$ was used, the pattern height was changed in order to vary the side wall area in that work. Therefore, it is hard to know that the demolding force mainly depends on the side wall area and/or the pattern height.

In this study, the demolding forces are examined by use of two molds whose pattern pitches and heights are different but side wall areas are same. The surface energy of the molds is always monitored and the relation between the demolding force and the mold surface energy is discussed.

\section{Experiments}

Si molds with line and space patterns (LS pattern) are fabricated on Si wafer of $10 \times 10 \mathrm{~mm}$. The pattern pitch is either $4 \mu \mathrm{m}$ or $0.4 \mu \mathrm{m}$ and the pattern region is $5 \times 5 \mathrm{~mm}$. The $4 \mu \mathrm{m}$ pitch pattern is fabricated by use of a conventional aligner. The $0.4 \mu \mathrm{m}$ pitch pattern is fabricated by use of laser interference patterning. The fourth harmonic of Nd:YAG laser, whose wave length is $\lambda=266$ $\mathrm{nm}$, is used. The $0.4 \mu \mathrm{m}$ pitch pattern can be obtained in the whole pattern area by one exposure of about $10 \mathrm{~s}$, although very long writing time is necessary when electron beam lithography is used. Cr pattern is fabricated on the $\mathrm{Si}$ wafer by the lift-off process and the $\mathrm{Si}$ wafer is etched by commercial ICP etcher (ULVAC, NE550). The

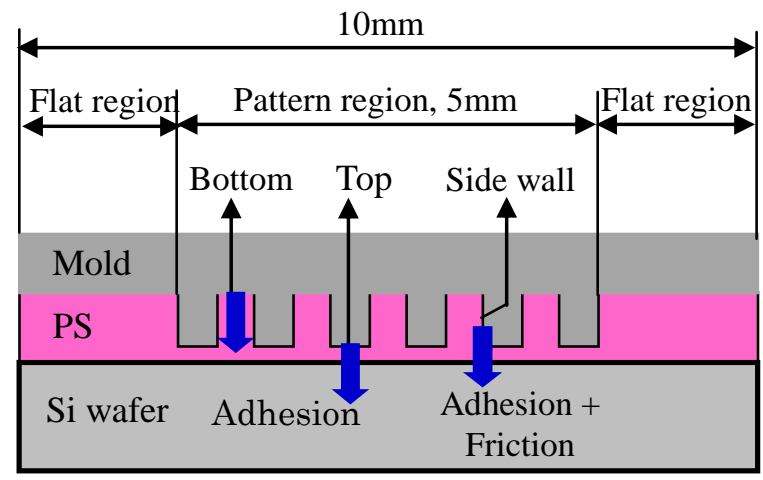

Fig. 1 Schematic view of mold and Si wafer 


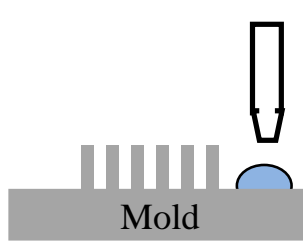

(a) Surface energy measurement before imprint

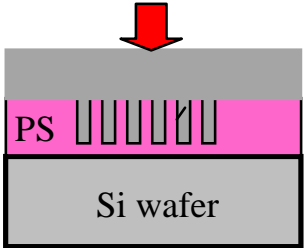

(b) Imprint

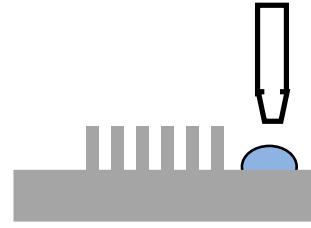

(c) Surface energy measurement after imprint

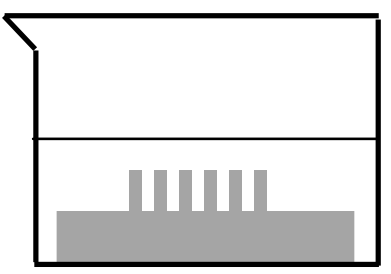

(d) Mold cleaning by toluen

Fig. 2 Illustration of process sequence

modified Bosch process is used in order to suppress the side wall roughness. The one cycle of the process consists of short deposition step by $\mathrm{C}_{4} \mathrm{~F}_{8}$ plasma $(\sim 5 \mathrm{~s})$ and etching step by $\mathrm{Ar}+\mathrm{SF}_{6}+\mathrm{CHF}_{3}$ plasma $(\sim 8 \mathrm{~s})$. The cycle is repeated and the desired depth cavity is fabricated. The details of the etching conditions were shown in the previous paper [5]. The molds with the $4 \mu \mathrm{m}$ and $0.4 \mu \mathrm{m}$ pitch LS patterns are named as the 4 $\mu \mathrm{m}$ mold and the $0.4 \mu \mathrm{m}$ mold, respectively. The schematic view of the mold is illustrated in Fig. 1. The mold size and its pattern area are $A_{S}=100 \mathrm{~mm}^{2}$ and $A_{P}=25 \mathrm{~mm}^{2}$, respectively. The pattern area is surrounded by the flat area. After the etching, the fabricated mold is bonded to a graphite mold holder of $15 \mathrm{~mm}$ in diameter. The standard anti-sticking treatment (Daikin: OPTOOL DSX) is carried out to the mold. In some experiments, the anti-sticking layer is degraded by the $\mathrm{UV} / \mathrm{O}_{3}$ treatment [6]. The mold surface energy is estimated from the contact angles for both pure water, $\theta_{\mathrm{H} 2 \mathrm{O}}$, and diiodomethane, $\theta_{\mathrm{CH} 212}[7,8]$. When the polar component of the surface energy is large, $\theta_{\mathrm{H} 20}$ becomes small. When the dispersive component is large, $\theta_{\mathrm{CH} 2 \mathrm{I} 2}$ becomes zmall. Yhe mold is pressed to $3.6 \mu \mathrm{m}$ thick polystyrene (PS) film on $\mathrm{Si}$ wafer of $30 \times 30 \mathrm{~mm}$. The molecular weight of the used PS is $350 \mathrm{k}$. The press conditions are $190{ }^{\circ} \mathrm{C}, 10 \mathrm{MPa}$ for $10 \mathrm{~min}$. The sample is cooled to the room temperature, and the press pressure is released. Finally, the $\mathrm{Si}$ wafer is removed from the mold. The pull force is applied to the mold. The direction of the pull force is vertical to the wafer surface [9]. The maximum pull force is defined as the demolding force. Several imprint experiments are repeated by one mold. The sequence of the experiment is shown in Fig. 2. (a) Before the imprint, the mold surface energy is measured. The contact angle is measured at the flat region of the mold. (b) The press and demolding processes are carried out. (c) After the imprint, the mold surface energy is measured. (d) The mold is always cleaned by toluene with an ultra sonic agitation for $5 \mathrm{~min}$. after one imprint process in order to remove PS resin which may adhere to the mold during the imprint process. In some experiments, $\mathrm{UV} / \mathrm{O}_{3}$ treatment is carried out in order to controll the mold surface energy.

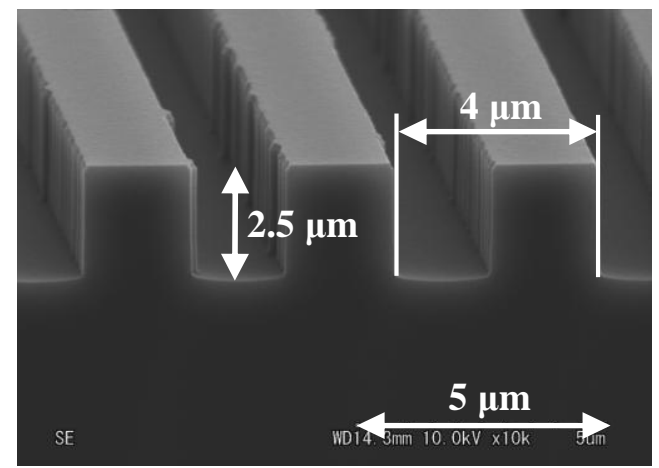

(a)

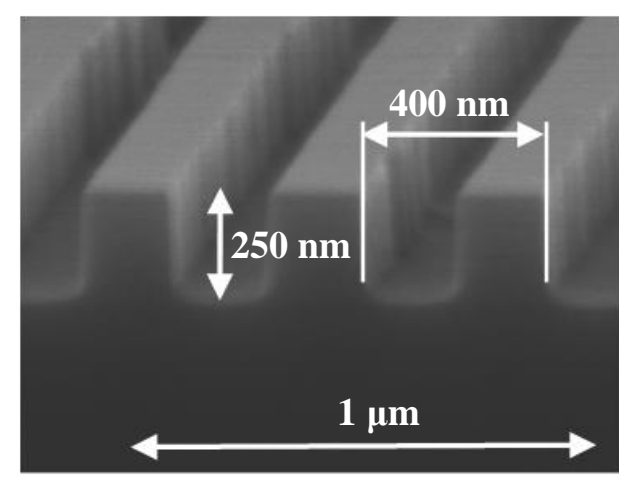

(b)

Fig. 3 SEM pictures of (a) $4 \mu \mathrm{m}$ mold and (b) $0.4 \mu \mathrm{m}$ mold. 


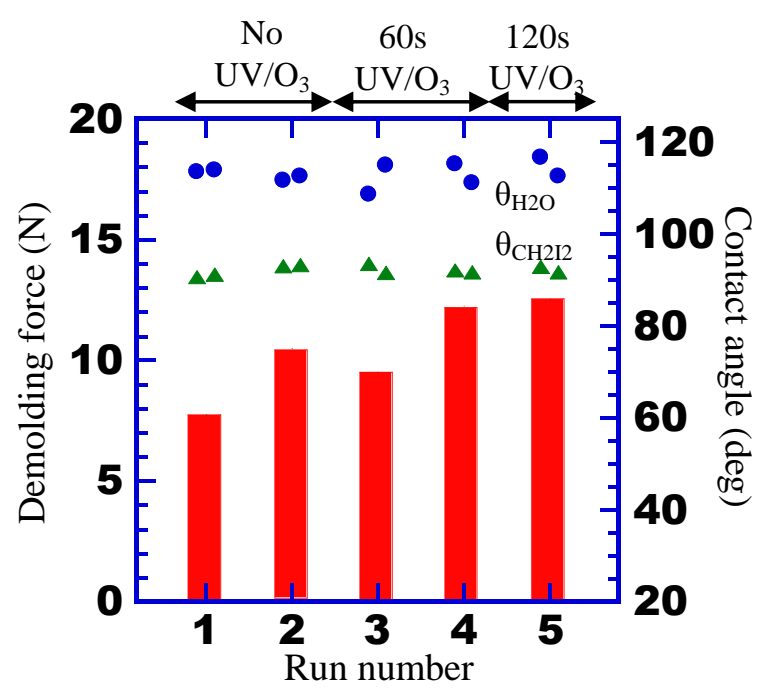

Fig. 4 Demolding force and contact angles for $4 \mu \mathrm{m}$ mold.

\section{Results}

\subsection{Fabricated molds and PS patterns}

The total side wall area, $A_{W}$, can be estimated by the following simple equation.

$$
A_{w}=\frac{2 H}{P} A_{P}
$$

where $\mathrm{H}$ and $\mathrm{P}$ are the pattern height and the pattern pitch, and $A_{P}$ is the pattern area. Since the values of $A_{P}$ are same for both the $4 \mu \mathrm{m}$ and 0.4 $\mu \mathrm{m}$ molds, the total side wall areas become same when the values of $\mathrm{H} / \mathrm{P}$ are same. The SEM pictures of the fabricated molds are shown in Fig.3. It is found that the molds with vertical side wall are obtained. For the $4 \mu \mathrm{m}$ mold, the pitch and pattern height are $4 \mu \mathrm{m}$ and $2.5 \mu \mathrm{m}$, respectively. The value of $\mathrm{H} / \mathrm{P}$ becomes 0.6 . For the $0.4 \mu \mathrm{m}$ mold, the pitch and pattern height are $0.4 \mu \mathrm{m}$ and $0.25 \mu \mathrm{m}$, respectively. The values of $\mathrm{H} / \mathrm{P}$ for both the molds become 0.6. The total side wall areas are same for both the molds. After the imprint, the heights of fabricated PS patterns are as high as the mold ones for both the molds. The mold cavities are completely filled by PS resin in the imprint process.

\subsection{Demolding force}

Figure 4 shows the demolding force for the 4 $\mu \mathrm{m}$ mold. The abscissa axis shows the run number. Bars show the demolding force for each run. Both $\theta_{\mathrm{H} 2 \mathrm{O}}$ and $\theta_{\mathrm{CH} 22}$ are also shown. The circle and triangle marks correspond to $\theta_{\mathrm{H} 2 \mathrm{O}}$ and $\theta_{\mathrm{CH} 212}$, respectively. For each run, two circle and triangle marks are plotted. The left and right ones show the contact angles before imprint and after imprint, respectively. The mold conditions are shown at the top of the figure. For the runs "1" and " 2 ", the

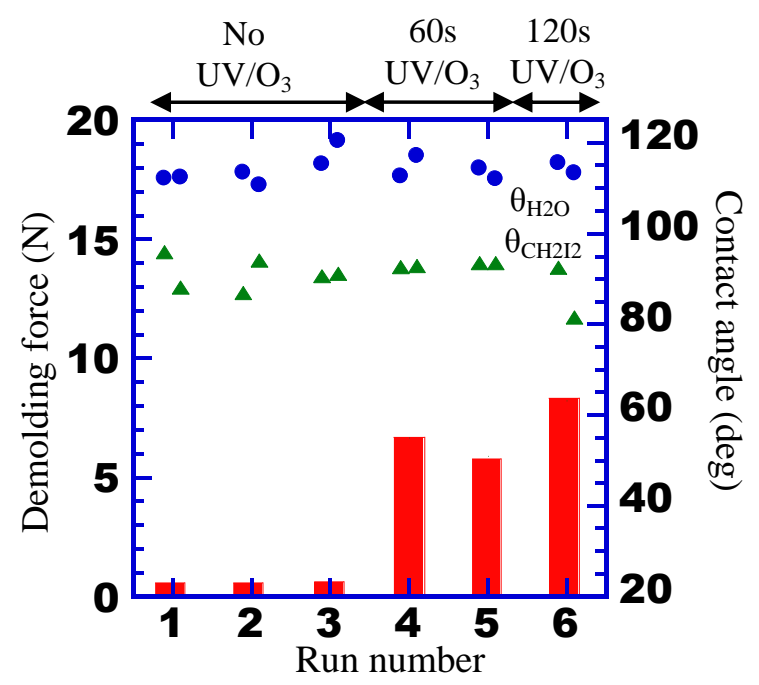

Fig. 5 Demolding force and contact angles for $0.4 \mu \mathrm{m}$ mold.

mold without $\mathrm{UV} / \mathrm{O}_{3}$ treatment is used. For the runs " 3 " and "4", the mold with the $\mathrm{UV} / \mathrm{O}_{3}$ treatment of $60 \mathrm{~s}$ is used. For the run " 5 ", the mold with the additional $\mathrm{UV} / \mathrm{O}_{3}$ treatment of $60 \mathrm{~s}$ is used. Then, the total time of the $\mathrm{UV} / \mathrm{O}_{3}$ treatment becomes $120 \mathrm{~s}$. The demolding force for the mold without the $\mathrm{UV} / \mathrm{O}_{3}$ treatment is about $9 \mathrm{~N}$. A small increase in the demolding force can be observed by the $\mathrm{UV} / \mathrm{O}_{3}$ treatment. Both $\theta_{\mathrm{H} 2 \mathrm{O}}$ and $\theta_{\mathrm{CH} 2 \mathrm{I2}}$ are almost same before and after the imprint. Moreover, they are almost same even when the $\mathrm{UV} / \mathrm{O}_{3}$ treatment is carried out. The degree of the anti-sticking layer degradation may be small through the experiments. Figure 5 shows the results for the $0.4 \mu \mathrm{m}$ mold. The runs " 1 ", " 2 " and " 3 " show the results for the $0.4 \mu \mathrm{m}$ mold without the $\mathrm{UV} / \mathrm{O}_{3}$ treatment. Their demolding forces are very small and the mold is released from the $\mathrm{Si}$ wafer during the sample setting to the demolding tool. Therefore, the demolding forces cannot be measured, but the values must be smaller than $1 \mathrm{~N}$. The demolding force for the $0.4 \mu \mathrm{m}$ mold abruptly increases by the first $\mathrm{UV} / \mathrm{O}_{3}$ treatment of $60 \mathrm{~s}$ and slightly increases by the additional $\mathrm{UV} / \mathrm{O}_{3}$ treatment of $60 \mathrm{~s}$. It is hard to observe the change in both $\theta_{\mathrm{H} 2 \mathrm{O}}$ and $\theta_{\mathrm{CH} 2 \mathrm{I2}}$ by the $\mathrm{UV} / \mathrm{O}_{3}$ treatment.

\section{Discusions}

When no $\mathrm{UV} / \mathrm{O}_{3}$ treatment is carried out, the mold has good anti-sticking layer. When the molds with good anti-sticking layer are used, the demolding force for the $0.4 \mu \mathrm{m}$ mold is much smaller than that for the $4 \mu \mathrm{m}$ mold even though their side wall areas are same. The pattern height rather than the side wall area is very important 


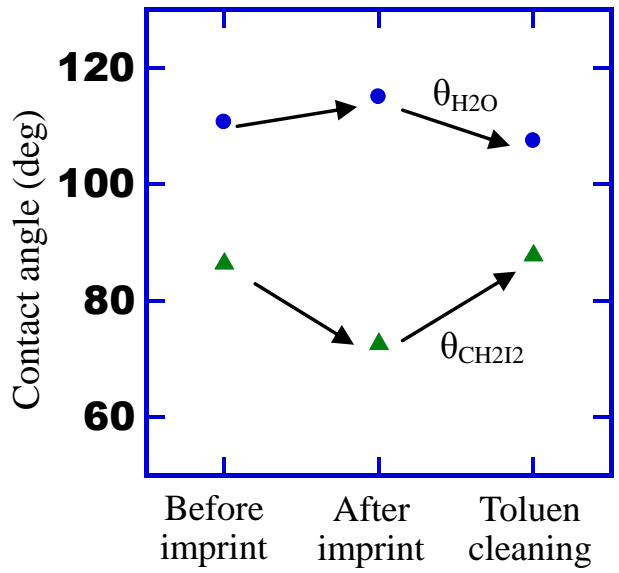

Fig.6 Variation in contact angles during one sequence

factor for the demolding force. By the $\mathrm{UV} / \mathrm{O}_{3}$ treatment, the increase of the demolding force for the $0.4 \mu \mathrm{m}$ mold is more obvious than that for the $4 \mu \mathrm{m}$ mold. The weak bond of anti-sticking layer must be removed by the first $\mathrm{UV} / \mathrm{O}_{3}$ treatment. For the demolding force, the degradation of the anti-sticking layer in the pattern region is more serious than that in the flat region because the side wall of the mold pattern is the source of the demolding force. The pattern density of the $0.4 \mu \mathrm{m}$ mold is larger than that of the $4 \mu \mathrm{m}$ mold. Therefore, more defects of the anti-sticking layer may be produced in the pattern region of the 0.4 $\mu \mathrm{m}$ mold than the $4 \mu \mathrm{m}$ mold. For the additional $\mathrm{UV} / \mathrm{O}_{3}$ treatment of $60 \mathrm{~s}$, the degree of anti-sticking layer degradation becomes small, because the weak anti-sticking layer has been removed by the first $\mathrm{UV} / \mathrm{O}_{3}$ treatment,

When the anti-sticking treatment is poor, resist resin sometimes adheres to a mold. An example of the detection method of the resist resin sticking is shown below. Figure 6 shows the changes in $\theta_{\mathrm{H} 2 \mathrm{O}}$ and $\theta_{\mathrm{CH} 212}$ during one process sequence of Fig. 2 for the flat mold. In this case, the demolding force is $15 \mathrm{~N}$. Since the demolding force is large, the anti-sticking treatment must be poor. The values of $\theta_{\mathrm{H} 2 \mathrm{O}}$ are about $110^{\circ}$ through the sequence. It means that the polar component of the surface energy is small. On the other hand, the value of $\theta_{\mathrm{CH} 212}$ decreases by the imprint process and recovers to the value before the imprint by the toluene cleaning. The measured values of $\theta_{\mathrm{H} 2 \mathrm{O}}$ and $\theta_{\mathrm{CH} 2 \mathrm{I2}}$ for the PS film are $102^{\circ}$ and $24^{\circ}$, respectively. For the PS and the mold surfaces, the values of $\theta_{\mathrm{CH} 2 \mathrm{I2}}$ are different but those of $\theta_{\mathrm{H} 2 \mathrm{O}}$ are same because the polar components of surface energy for both the surfaces are small. When PS resin adheres to the mold surface, the change in $\theta_{\mathrm{CH} 212}$ can be detected but it is hard to detect the change in $\theta_{\mathrm{H} 2 \mathrm{O}}$.

\section{Conclusions}

Two molds with $0.4 \mu \mathrm{m}$ and $4 \mu \mathrm{m}$ pitch LS patterns are fabricated. The pattern heights are adjusted in order to keep the side wall areas of the both molds same. The demolding force for the 0.4 $\mu \mathrm{m}$ mold is much smaller than that for the $4 \mu \mathrm{m}$ mold. The pattern height rather than the side wall area is important factor for the demolding force. The demolding force is increased by the $\mathrm{UV} / \mathrm{O}_{3}$ treatment because of the degradation of the anti-sticking layer. The effect is more obvious for the $0.4 \mu \mathrm{m}$ mold than for the $4 \mu \mathrm{m}$ mold, because the pattern density of the $0.4 \mu \mathrm{m}$ mold is larger than that of the $4 \mu \mathrm{m}$ mold.

\section{References}

1. S. Y. Chou, P. R. Krauss, and P. J. Renstrom, Appl. Phys. Lett. 67 (1995) 3114.

2. J. Taniguchi, T. Kawasaki, Y. Tokano, Y. Kogo, I. Miyamoto, M. Komuro, H. Hiroshima, N. Sakai, and K. Tada: Jpn. J. Appl. Phys. 41 (2002) 4194.

3. V. Trabadelo, H. Schift, S. Merino, S. Bellini, J. Gobrecht: Microelectron. Eng., 85 (2008) 907.

4. H. Kawata, K. Kubo, M. Matsue, M. Yasuda and Y. Hirai, MNE2009 (Ghent, Belgium), O-Litho-26.

5. H. Kawata, M. Matsue, K. Kubo, M. Yasuda, and Y. Hirai: Microelectron. Eng. 86 (2009) 700

6. J. Ishihara, A. Koszewsk, M. Kayama, H. Kawata, Z. Rymuza, Y. Hirai, MNC2007 (Kyoto, Japan) 7B-7-6.

7. F. M. Fowkes, J. Phys. Chem., 66 (1962) 382.

8. D. K. Owens and R. C. Wendt, J. Appl. Polymer Sci., 13 (1969) 1941.

9. H. Kawata, Y. Watanabe, N. Fujikawa, M. Yasuda and Y. Hirai, J. Vac. Sci. Technol. B, 28, (2010) C6M77 